\title{
BMJ Open High TG/HDL ratio suggests a higher risk of metabolic syndrome among an elderly Chinese population: a cross- sectional study
}

\author{
Guqiao Nie (D) , ${ }^{1}$ Shukai Hou, ${ }^{2}$ Meng Zhang, ${ }^{3}$ Wen Peng (D) ${ }^{4}$
}

To cite: Nie G, Hou S, Zhang M, et al. High $\mathrm{TG} / \mathrm{HDL}$ ratio suggests a higher risk of metabolic syndrome among an elderly Chinese population: a cross-sectional study. BMJ Open 2021;11:e041519. doi:10.1136/ bmjopen-2020-041519

- Prepublication history for this paper is available online. To view these files, please visit the journal online (http://dx.doi. org/10.1136/bmjopen-2020041519).

Received 12 June 2020

Revised 26 December 2020 Accepted 26 February 2021

Check for updates

(c) Author(s) (or their employer(s)) 2021. Re-use permitted under CC BY-NC. No commercial re-use. See rights and permissions. Published by BMJ.

${ }^{1}$ Department of Geriatrics, Union Hospital, Tongji Medical College, Huazhong University of Science and Technology, WuHan, Hubei, China

${ }^{2}$ Community health service center, Gutian street, Qiaokou District, WuHan, HuBei, China ${ }^{3}$ Department of General practice, Union Hospital, Tongji Medical

College, Huazhong University of Science and Technology, WuHan, HuBei, China

${ }^{4}$ Department of General Practice, Union Hospital, Tongji Medical College, Huazhong University of Science and Technology, WuHan, Hubei, China

Correspondence to

Professor Wen Peng;

pengwen666@sina.com

\section{ABSTRACT}

Objectives To investigate the relationship between triglyceride/high-density lipoprotein cholesterol (TG/HDL-C) ratio and metabolic syndrome in the elderly population of China, and to determine the best critical value of TG/HDL-C in higher risk of metabolic syndrome in this population.

Design Cross-sectional study.

Setting Our study was conducted in a community physical examination centre in Wuhan, China between 1 January 2016 and 31 December 2016.

Participants The physical examination data from 1267 elderly people (aged over 65 years) in the community were analysed in this study. The average age of the study participants was $71.64 \pm 5.605$ years.

Primary outcome measures Correlation between the TG/ HDL-C ratio and metabolic syndrome; the optimum cutoff of the TG/HDL-C ratio for the prediction of metabolic syndrome.

Results The $\mathrm{TG} / \mathrm{HDL}-\mathrm{C}$ ratio showed a significant positive correlation with metabolic syndrome $(r=0.420, p<0.001)$ in the elderly Chinese population. Binary logistic regression analysis showed that the TG/HDL-C ratio was an independent risk factor for metabolic syndrome $(\mathrm{OR}=3.07$ (95\% Cl: 2.402 to 3.924), $p<0.001$ ) after adjusting for blood pressure, blood glucose, age, sex and body mass index. The receiver operating characteristic curves of TG/HDL-C ratio and metabolic syndrome showed that in the elderly population, a TG/HDL-C ratio of 1.49 can be used as the critical value for a higher risk of metabolic syndrome. At this value, the specificity and sensitivity of the measure were optimal $(80.8 \%$ and $72.4 \%$, respectively).

Conclusion In this study, we found a significant correlation between $\mathrm{TG} / \mathrm{HDL}-\mathrm{C}$ ratio and metabolic syndrome. And high TG/HDL ratio suggests a higher risk of metabolic syndrome among an elderly Chinese population.

\section{INTRODUCTION}

Metabolic syndrome (MetS) refers to a pathological state involving the impaired metabolism of proteins, fats, carbohydrates and other substances in the human body. It is a complex group of metabolic disorder syndromes, including risk factors associated with cardiovascular disease (CVD) and diabetes. $^{12}$ The prevalence of MetS is high

\section{Strengths and limitations of this study}

- The study sample had a wide age range (65-93 years), and the study included information on their basic characteristics like sex, waist circumference, marital status, diagnosis of hypertension and so on.

- This study is the first to explore the correlation between the triglyceride/high-density lipoprotein cholesterol (TG/HDL-C) ratio and metabolic syndrome among elderly Chinese people.

- Our study is a cross-sectional study, which cannot reflect the causal relationship between TG/HDL and metabolic syndrome.

- Our study did not adjust for possible confounders such as exercise and lipid-regulating drugs.

The study population came from only one community.

and rapidly increasing among the elderly population in China. ${ }^{3}$ Risk factors include central obesity, increased blood pressure, increased fasting blood glucose (FBG) levels and abnormal blood lipids. The prevalence of cardiovascular events and the risk of mortality are higher among patients with MetS than among the general population. ${ }^{4}$ Therefore, the early diagnosis of MetS and timely intervention can prevent the unnecessary complications related to its progression and help reduce the risk of CVD caused by metabolic disorders. From a clinical perspective, individuals can benefit from the establishment of appropriate diagnostic methods and criteria that can accurately distinguish patients with MetS from healthy people.

This study aimed to explore the correlation between the ratio of triglycerides to highdensity lipoprotein cholesterol in the blood (TG/HDL-C) and MetS in the elderly Chinese population, calculate the cut-off value of TG/ HDL-C for higher risk of MetS in this population and determine the optimal critical value for higher risk of MetS, in order to provide a theoretical basis for the early identification 
and management of elderly patients with MetS, as well as prevent the development of cardiovascular and cerebrovascular diseases.

\section{PARTICIPANTS AND METHODS}

\section{Study population}

This was a cross-sectional study, involving data from 1267 elderly individuals examined and interviewed at a community physical examination centre, between 1 January 2016 and 31 December 2016.

Participants' data were included in this study if the patient met all of the following inclusion criteria: lived in China, aged $\geq 65$ years, provided basic demographic information, provided accurate biochemical measurements and consented to a full clinical examination.

Participants were excluded if they met one or more of the following exclusion criteria: incomplete data provided; presence of one of the following conditions: physical disability, acute infection, acute myocardial infarction, acute cerebral infarction, renal failure, dialysis or active stage malignancies; or use of the following medications: hormone replacement therapy and oral or injectable glucocorticoids.

\section{Clinical characteristics}

Data for this study were obtained from the physical examination records of the elderly people in the community. General demographic and clinical data such as age, sex, marital status, height, weight, waist circumference (WC) and history of hypertension were obtained through oral interview. The doctor used a mercury sphygmomanometer on the upper arm to measure blood pressure-systolic blood pressure (SBP) and diastolic blood pressure (DBP). The average value of two blood pressure measurements taken at least 5 min apart was used.

\section{Biochemical parameters}

Biochemical indices including FBG, blood lipids and uric acid (UA) were all measured using venous blood obtained from the participants on an empty stomach. Levels of serum UA, TGs, HDL-C and low-density lipoprotein cholesterol and FBG were measured using Roche E602 and Roche C701 (both of which are automatic biochemical analysers).

\section{Definition of MetS in this study}

The diagnosis of MetS in this study was made according to the following definition provided by the Chinese Diabetes Society:

1. Overweight and/or obese: body mass index (BMI) $\geq 25 \mathrm{~kg} / \mathrm{m}^{2}$.

2. Hyperglycaemia: $\mathrm{FBG} \geq 6.1 \mathrm{mmol} / \mathrm{L} \quad(110 \mathrm{mg} / \mathrm{dL})$, and/or 2-hour plasma glucose $\geq 7.8 \mathrm{mmol} / \mathrm{L}$ (140 mg/ $\mathrm{dL}$ ), and/or people with diagnosed and treated diabetes.

3. Hypertension: SBP/DBP $\geq 140 / 90 \mathrm{~mm} \mathrm{Hg}$ and/or with diagnosed and treated hypertension.
4. Dyslipidaemia: fasting TGs $\geq 1.7 \mathrm{mmol} / \mathrm{L}(150 \mathrm{mg} / \mathrm{dL})$ and/or fasting blood HDL-C $<0.9 \mathrm{mmol} / \mathrm{L}(35 \mathrm{mg}$ / dL) (among men) and $<1.0 \mathrm{mmol} / \mathrm{L} \quad(39 \mathrm{mg} / \mathrm{dL})$ (among women).

MetS was diagnosed if at least three of the above four components were present.

\section{Participant and public involvement}

Participants were not involved in the design or conduct of the study. It is an anonymous, non-invasive examination, only oral notification, waiving the signing of written informed consent.

\section{Statistical analyses}

First, the data were divided into two groups according to the diagnosis of MetS, MetS group and non-MetS group. The percentages of participants in the above two groups who were positive for each component were reported and compared using the $\mathrm{X}^{2}$ test. The mean value $\pm \mathrm{SD}$ was calculated for each of the continuous variables and compared using independent-samples t-tests, and other categorical variables were compared using the $\mathrm{X}^{2}$ test. The Kendall's tau-b and Spearman's rank correlation coefficients were used to analyse the correlation between MetS and the TG/HDL-C ratio, UA, BMI, WC, age, sex and education. Binary logistic regression was used to analyse risk factors, and the results for each risk factor were described in terms of the non-standardised coefficient B, OR, its $95 \% \mathrm{CI}$ and $\mathrm{p}$ value. A receiver operating characteristic (ROC) curve of MetS versus TG/HDL-C ratio was drawn to determine the optimum cut-off point for MetS diagnosis.

\section{RESULTS}

\section{Clinical characteristics}

The characteristics of the 1267 participants included in this study are shown in table 1 . The mean age of the participants was $71.64 \pm 5.605$ years. Participants diagnosed with MetS (MetS group) have statistically significant differences in the TG/HDL-C ratio, age, blood pressure, WC, FBG, UA level, hypertension, TG and HDL-C compared with participants not diagnosed with MetS (non-MetS group).

\section{Bivariate correlation analyses between the covariates and MetS}

The results of the bivariate correlation analyses are shown in table 2. The TG/HDL-C ratio, UA, BMI, WC, FBG, TG, DBP, SBP and age were significantly correlated with MetS; however, gender, marital status, education and ECG results were not.

\section{Logistic regression analysis between $\mathrm{TG} / \mathrm{HDL}-\mathrm{C}$ ratio and MetS}

Model 1 shows the association between the TG/HDL-C ratio and MetS without adjusting for any confounders (table 3). The TG/HDL-C ratio is an independent risk factor for MetS. The unadjusted OR is 2.280 (95\% CI: 1.947 to 2.670$), \mathrm{p}<0.001$. After adjusting for potential 
Table 1 Clinical characteristics of the different groups

\begin{tabular}{|c|c|c|c|}
\hline & MetS ( $\mathrm{N}=234)$ & $\begin{array}{l}\text { Non-MetS } \\
(\mathrm{N}=1033)\end{array}$ & \\
\hline Characteristic or parameter & Mean \pm SD & Mean \pm SD & $P$ value \\
\hline BMI $\left(\mathrm{kg} / \mathrm{m}^{2}\right)$ & $27.14 \pm 3.19$ & $23.98 \pm 3.25$ & $<0.001$ \\
\hline DBP (mm Hg) & $82.97 \pm 10.97$ & $76.54 \pm 11.29$ & $<0.001$ \\
\hline TG/HDL-C & $2.07 \pm 1.09$ & $1.17 \pm 0.89$ & $<0.001$ \\
\hline $\mathrm{TC}$ (mmol/L) & $4.78 \pm 1.10$ & $4.60 \pm 0.89$ & 0.024 \\
\hline $\mathrm{HDL}$ (mmol/L) & $1.02 \pm 0.22$ & $2.75 \pm 0.77$ & $<0.001$ \\
\hline LDL (mmol/L) & $2.92 \pm 0.91$ & $2.76 \pm 0.76$ & 0.005 \\
\hline Gender (\% male) & $107(45.72)$ & $449(43.46)$ & 0.56 \\
\hline Hypertension & $205(87.61)$ & $332(32.14)$ & $<0.001$ \\
\hline Abnormal ECG & $148(63.25)$ & $615(59.54)$ & 0.302 \\
\hline
\end{tabular}

Variables are described in terms of either mean $\pm S D$ or percentage. The $p$ value was calculated using the Student's t-test or the $X^{2}$ test. $\mathrm{BMI}$, body mass index; DBP, diastolic blood pressure; FBG, fasting blood glucose; HDL-C, high-density lipoprotein cholesterol; LDL-C, lowdensity lipoprotein cholesterol; MetS, metabolic syndrome; SBP, systolic blood pressure; TC, total cholesterol; TG, triglyceride; UA, uric acid.

\begin{tabular}{lll}
\hline $\begin{array}{l}\text { Table } 2 \text { Correlations of patient characteristics with } \\
\text { diagnosis of metabolic syndrome }\end{array}$ & \multicolumn{1}{l}{} \\
\hline Characteristic or parameter & $\mathbf{r}$ & P value \\
\hline TG/HDL-C & 0.42 & $<0.001$ \\
\hline UA (mmol/L) & 0.189 & $<0.001$ \\
\hline BMI (kg/m²) & 0.363 & $<0.001$ \\
\hline Waist circumference & 0.308 & $<0.001$ \\
\hline FBG (mmol/L) & 0.364 & $<0.001$ \\
\hline LDL-C (mmol/L) & 0.059 & 0.037 \\
\hline HDL-C (mmol/L) & -0.301 & $<0.001$ \\
\hline TC (mmol/L) & 0.057 & 0.044 \\
\hline TG (mmol/L) & 0.383 & $<0.001$ \\
\hline Gender & 0.018 & 0.53 \\
\hline ECG & 0.03 & 0.286 \\
\hline Age (years) & -0.012 & 0.672 \\
\hline Education & -0.012 & 0.647 \\
\hline Hypertension & 0.436 & $<0.001$ \\
\hline Marital status & -0.01 & 0.712 \\
\hline DBP (mm Hg) & 0.217 & $<0.001$ \\
\hline SBP (mm Hg) & 0.381 & $<0.001$ \\
\hline
\end{tabular}

$\mathrm{R}=$ Kendall's tau-b correlation coefficient or Spearman's rank correlation coefficient.

BMI, body mass index; DBP, diastolic blood pressure; FBG, fasting blood glucose; HDL-C, high-density lipoprotein cholesterol; LDL-C, low-density lipoprotein cholesterol; SBP, systolic blood pressure; TC, total cholesterol; TG, triglyceride; UA, uric acid. confounders, we found that the TG/HDL-C ratio is still an independent risk factor for MetS (OR=2.301 (95\% CI: 1.884 to 2.811$), \mathrm{p}<0.001$ ).

\section{ROC curve}

By drawing ROC curves, we found the best critical value is 1.49 ; the specificity and sensitivity were optimal $(80.8 \%$ and $72.4 \%$, respectively).

As shown in figure 1, the horizontal axis represents one minus the specificity, and the vertical axis represents sensitivity. The area under the curve (AUC) for the TG versus HDL-C graph was 0.813 (95\% CI: 0.784 to 0.842 ), with a statistically significant prediction effect $(\mathrm{p}<0.001)$. The Jordan index is 0.532 , which also indicates that TG/ HDL ratio has a good effect in higher risk of MetS. In addition, in view of the influence of gender in MetS, we drew ROC curves for men and women. The best cut-off values are 1.437 and 1.196 , respectively. The sensitivity and specificity are $74.8 \%$ and $78.4 \%$ for men, and $86.4 \%$ and $67.4 \%$ for women. Finally, we use TG and HDL to draw the ROC curve separately, and we can see that the predicted value of both is lower than the ratio of the two as the figure 1 shows (the AUCs of TG and HDL are 0.786 and 0.276 , respectively).

\section{DISCUSSION}

In this study, we verified the correlation between the TG/HDL-C ratio and MetS, and our results established 


\begin{tabular}{|c|c|c|c|c|}
\hline Model & Exposure & B & $P$ value & OR $(95 \% \mathrm{CI})$ \\
\hline Model 1 & TG/HDL-C & 0.824 & $<0.001$ & 2.280 (1.947 to 2.670$)$ \\
\hline \multirow[t]{6}{*}{ Model 2} & TG/HDL & 0.842 & $<0.001$ & $2.321(1.903$ to 2.831$)$ \\
\hline & FBG & 0.616 & $<0.001$ & 1.852 (1.638 to 2.095 ) \\
\hline & $\mathrm{BMI}$ & 0.259 & $<0.001$ & $1.295(1.218$ to 1.377$)$ \\
\hline & UA & 0.003 & 0.009 & $1.003(1.001$ to 1.005$)$ \\
\hline & SBP & 0.056 & $<0.001$ & $1.058(1.044$ to 1.072$)$ \\
\hline & DBP & 0.023 & 0.019 & $1.023(1.004$ to 1.043$)$ \\
\hline \multirow[t]{8}{*}{ Model 3} & $\mathrm{TG} / \mathrm{HDL}$ & 0.834 & $<0.001$ & 2.301 (1.884 to 2.811 ) \\
\hline & FBG & 0.616 & $<0.001$ & 1.851 (1.636 to 2.094$)$ \\
\hline & $\mathrm{BMI}$ & 0.255 & $<0.001$ & $1.29(1.212$ to 1.374$)$ \\
\hline & UA & 0.003 & 0.009 & $1.003(1.001$ to 1.005$)$ \\
\hline & SBP & 0.058 & $<0.001$ & 1.06 (1.046 to 1.074$)$ \\
\hline & DBP & 0.021 & 0.039 & 1.021 (1.001 to 1.041$)$ \\
\hline & Gender & -0.038 & 0.858 & 0.963 (0.635 to 1.459$)$ \\
\hline & Age & -0.023 & 0.219 & 0.977 (0.942 to 1.014$)$ \\
\hline
\end{tabular}

Model 1: crude model; model 2: adjusted for UA, BMI, FBG, SBP, DBP; model 3: adjusted for age, gender, UA, BMI, FBG, SBP, DBP. BMI, body mass index; DBP, diastolic blood pressure; FBG, fasting blood glucose; HDL-C, high-density lipoprotein cholesterol; MetS, metabolic syndrome; SBP, systolic blood pressure; TG, triglyceride; UA, uric acid.

the accuracy of the TG/HDL-C ratio as a diagnostic for higher risk of MetS among the elderly people in China.

In MetS, TG and HDL are part of the diagnostic criteria, but the International Diabetes Association does not include TG/HDL-C ratio in the diagnosis of MetS. ${ }^{5}$ However, TG/HDL-C ratio seems to play an important role in metabolic disorders. ${ }^{6}$ Large-scale prospective studies show that the TC/HDL-C ratio can be used as a reliable index to predict coronary heart disease and death. ${ }^{7-14}$ A large number of studies have shown that TG/ HDL-C can be used as a simple alternative indicator of
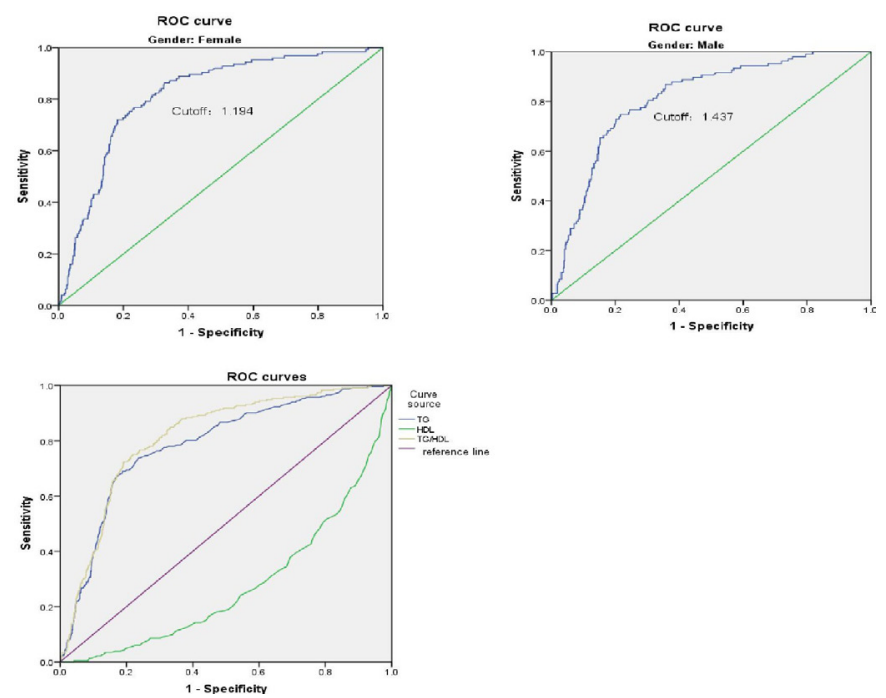

Figure 1 Receiver operating characteristic (ROC) curve of the TG/HDL-C ratio as a high-risk indicator of metabolic syndrome. HDL-C, high-density lipoprotein cholesterol; TG, triglyceride. insulin resistance. ${ }^{15-17}$ The National Cholesterol Education Program Adult Treatment Panel III is the commonly used diagnostic standard of MetS in the world. It considers insulin resistance, hypertension, atherosclerosis and abdominal obesity as diagnostic criteria, ${ }^{18}$ which indirectly shows that TG/HDL-C is feasible to predict MetS. Recently, the TC/HDL-C ratio has been proposed as an alternative method to diagnose MetS. In a study of children and adolescents with obesity, the TG/HDL-C ratio had high sensitivity $(80 \%)$ and specificity $(75 \%)$ to MetS, with a cut-off point of $1.25{ }^{6}$ In our study on an elderly population, a TG/HDL-C ratio of 1.49 was found to be the best critical value. Clinical studies have shown that the TG/HDL-C ratio can be used to predict MetS and to assess the risk of cardiovascular events in older people. ${ }^{18-20}$ Most studies are limited to specific populations and races. For example, the TG/HDL-C ratio has been found to have a high predictive value for MetS among Korean adolescents. ${ }^{21}$ Prior to our study, research on the link between TG/HDL-C and MetS in the Chinese elderly was rare. Additionally, many clinical trials to date have shown that lipid metabolism disorder has toxic effects on cells, which can affect the function of islet $\beta$ cells and cause or aggravate insulin resistance. ${ }^{22}$ Insulin resistance and deficiency of insulin secretion can further aggravate lipid metabolism disorders. ${ }^{21} 2324$ In the future, studies can carefully investigate the effectiveness of the TG/HDL-C ratio in the diagnosis of insulin resistance since these measurements are usually easier to obtain than performing the insulin resistance test.

In our study, UA also showed a significant positive correlation with MetS. Experimental studies have shown 
that UA can penetrate smooth muscle fibres through the organic anion transport system, thereby activating various transmission pathways and increasing the expression of inflammatory mediators. ${ }^{25}{ }^{26}$ Therefore, further research into the relationship between UA and MetS is needed in the future. After consulting the literature, we have studies suggesting that there is a correlation between UA, age, sex and MetS, ${ }^{27-29}$ and our research has also found this. In order to eliminate these variables known to be associated with MetS, we performed a multiple logistic regression analysis to eliminate the interference of these confounding factors.

Our study found that with an increase in the TG/ HDL-C ratio, there was a gradual increase in the number of people diagnosed with MetS. When TG/HDL is greater than 1.437 for men and 1.194 for women, it indicates that the patient is at a high risk of MetS. At the same time, we need to attach great importance to the patient's UA, fasting blood sugar and blood pressure. The early detection of patients at a high risk for MetS through the measurement of the TG/HDL-C ratio can help to actively prevent or treat them before disease symptoms appear, thus providing patients with the greatest clinical benefit.

\section{Limitations}

The study participants all come from one community in China; therefore, the sample may not be representative of the elderly population in China. We recruited only those who had completed the comprehensive health examination, which may have biased our main findings. Our study is a cross-sectional study, which cannot reflect the causal relationship between TG/HDL and MetS. In addition, our analysis adjusted for age, sex and so on, but not for physical exercise and diet that are known to affect blood lipids. We expect more research to be done in the future based on this study.

\section{CONCLUSIONS}

We found a strong correlation between the TG/HDL-C ratio and MetS. Elderly Chinese people with a high TG/ HDL-C ratio are at high risk of MetS. In our study sample, a TG/HDL-C ratio of 1.49 was found to be the optimal critical value. This study is of great significance for the early identification and management of MetS among the elderly people in China. The measurement of the TG/HDL-C ratio can help clinicians to identify elderly people who need targeted treatment, management and follow-up.

Acknowledgements The authors would like to thank the participants for their contribution to this study.

Contributors Study concept and design — GN and WP. Data acquisition—GN and KSH. Data analyses - GN, MZ and WP. Data interpretation-WP and GN. All authors contributed to writing, revising and approving the final manuscript.

Funding The authors have not declared a specific grant for this research from any funding agency in the public, commercial or not-for-profit sectors.

Competing interests None declared.
Patient and public involvement Patients and/or the public were not involved in the design, or conduct, or reporting, or dissemination plans of this research.

Patient consent for publication Not required.

Ethics approval This study was conducted in accordance with the contents of the Declaration of Helsinki. Since this study included identified data, participants were not required to provide informed consent. The study was approved by the Institutional Review Board of Tongji Medical College, Huazhong University of Science and Technology (S273).

Provenance and peer review Not commissioned; externally peer reviewed.

Data availability statement № data are available.

Open access This is an open access article distributed in accordance with the Creative Commons Attribution Non Commercial (CC BY-NC 4.0) license, which permits others to distribute, remix, adapt, build upon this work non-commercially, and license their derivative works on different terms, provided the original work is properly cited, appropriate credit is given, any changes made indicated, and the use is non-commercial. See: http://creativecommons.org/licenses/by-nc/4.0/.

\section{ORCID iDs}

Guqiao Nie http://orcid.org/0000-0002-5467-6431

Wen Peng http://orcid.org/0000-0003-0566-0801

\section{REFERENCES}

1 Eckel RH, Grundy SM, Zimmet PZ. The metabolic syndrome. Lancet 2005;365:1415-28.

2 Kassi E, Pervanidou P, Kaltsas G, et al. Metabolic syndrome: definitions and controversies. BMC Med 2011;9:48.

3 Liu M, Wang J, Jiang B, et al. Increasing prevalence of metabolic syndrome in a Chinese elderly population: 2001-2010. PLoS One 2013;8:e66233.

4 Vega GL, Barlow CE, Grundy SM, et al. Triglyceride-to-high-densitylipoprotein-cholesterol ratio is an index of heart disease mortality and of incidence of type 2 diabetes mellitus in men. J Investig Med 2014;62:345-9.

5 Wittcopp C, Conroy R. Metabolic syndrome in children and adolescents. Pediatr Rev 2016;37:193-202.

6 Liang J, Fu J, Jiang Y, et al. Triglycerides and high-density lipoprotein cholesterol ratio compared with homeostasis model assessment insulin resistance indexes in screening for metabolic syndrome in the Chinese obese children: a cross section study. BMC Pediatr 2015;15:138.

7 Packard CJ, Shepherd J. Lipoprotein heterogeneity and apolipoprotein B metabolism. Arterioscler Thromb Vasc Biol 1997;17:3542-56.

8 McLaughlin T, Abbasi F, Cheal K, et al. Use of metabolic markers to identify overweight individuals who are insulin resistant. Ann Intern Med 2003;139:802-9.

9 Giannini C, Santoro N, Caprio S, et al. The triglyceride-to-HDL cholesterol ratio: association with insulin resistance in obese youths of different ethnic backgrounds. Diabetes Care 2011;34:1869-74.

10 Grover SA, Palmer CS, Coupal L. Serum lipid screening to identify high-risk individuals for coronary death. The results of the lipid research clinics prevalence cohort. Arch Intern Med 1994;154:679-84.

11 Maruyama C, Imamura K, Teramoto T. Assessment of LDL particle size by triglyceride/HDL-cholesterol ratio in non-diabetic, healthy subjects without prominent hyperlipidemia. J Atheroscler Thromb 2003;10:186-91.

12 Pacifico L, Bonci E, Andreoli G, et al. Association of serum triglyceride-to-HDL cholesterol ratio with carotid artery intimamedia thickness, insulin resistance and nonalcoholic fatty liver disease in children and adolescents. Nutr Metab Cardiovasc Dis 2014;24:737-43.

13 Ju S-Y, Lee J-Y, Kim D-H. Association of metabolic syndrome and its components with all-cause and cardiovascular mortality in the elderly: a meta-analysis of prospective cohort studies. Medicine 2017;96:e8491.

14 Castelli WP, Garrison RJ, Wilson PW, et al. Incidence of coronary heart disease and lipoprotein cholesterol levels. The Framingham study. JAMA 1986;256:2835-8.

15 Geloneze B, Vasques ACJ, Stabe CFC, et al. HOMA1-IR and HOMA2-IR indexes in identifying insulin resistance and metabolic syndrome: Brazilian metabolic syndrome study (BRAMS). Arq Bras Endocrinol Metabol 2009;53:281-7. 
16 Galgani JE, Moro C, Ravussin E. Metabolic flexibility and insulin resistance. Am J Physiol Endocrinol Metab 2008;295:E1009-17.

17 Ren X, Chen ZA, Zheng S, et al. Association between triglyceride to $\mathrm{HDL}-\mathrm{C}$ ratio $(\mathrm{TG} / \mathrm{HDL}-\mathrm{C})$ and insulin resistance in Chinese patients with newly diagnosed type 2 diabetes mellitus. PLoS One 2016;11:e0154345.

18 Salazar MR, Carbajal HA, Espeche WG, et al. Identification of cardiometabolic risk: visceral adiposity index versus triglyceride/HDL cholesterol ratio. Am J Med 2014;127:152-7.

19 da Luz PL, Cesena FHY, Favarato D, et al. Comparison of serum lipid values in patients with coronary artery disease at $<50,50$ to 59,60 to 69 , and $>70$ years of age. Am J Cardiol 2005;96:1640-3.

20 Pérez-Martínez P, Mikhailidis DP, Athyros VG, et al. Lifestyle recommendations for the prevention and management of metabolic syndrome: an international panel recommendation. Nutr Rev 2017;75:307-26.

21 Chu S-Y, Jung J-H, Park M-J, et al. Risk assessment of metabolic syndrome in adolescents using the triglyceride/high-density lipoprotein cholesterol ratio and the total cholesterol/high-density lipoprotein cholesterol ratio. Ann Pediatr Endocrinol Metab 2019;24:41-8

22 Bjornstad P, Eckel RH. Pathogenesis of lipid disorders in insulin resistance: a brief review. Curr Diab Rep 2018;18:127.
23 Chen Q-J, Lai H-M, Chen B-D, et al. Appropriate LDL-C-to-HDL-C ratio cutoffs for categorization of cardiovascular disease risk factors among Uygur adults in Xinjiang, China. Int J Environ Res Public Health 2016:13:235

24 Yeh W-C, Tsao Y-C, Li W-C, et al. Elevated triglyceride-to-HDL cholesterol ratio is an indicator for insulin resistance in middle-aged and elderly Taiwanese population: a cross-sectional study. Lipids Health Dis 2019;18:176.

25 Soltani Z, Rasheed K, Kapusta DR, et al. Potential role of uric acid in metabolic syndrome, hypertension, kidney injury, and cardiovascular diseases: is it time for reappraisal? Curr Hypertens Rep 2013;15:175-81.

26 Mukhopadhyay P, Ghosh S, Pandit K, et al. Uric acid and its correlation with various metabolic parameters: a population-based study. Indian J Endocrinol Metab 2019;23:134-9.

27 King C, Lanaspa MA, Jensen T, et al. Uric acid as a cause of the metabolic syndrome. Contrib Nephrol 2018;192:88-102.

28 Pucci G, Alcidi R, Tap L, et al. Sex- and gender-related prevalence, cardiovascular risk and therapeutic approach in metabolic syndrome: a review of the literature. Pharmacol Res 2017;120:34-42.

29 Kanbay M, Jensen T, Solak Y, et al. Uric acid in metabolic syndrome: from an innocent bystander to a central player. Eur $J$ Intern Med 2016;29:3-8 